\title{
Structure and receptor-binding properties of an airborne transmissible avian influenza $A$ virus hemagglutinin H5 (VN1203mut)
}

\author{
Xishan $\mathrm{Lu}^{1,3}$, Yi Shi ${ }^{2}$, Wei Zhang ${ }^{3,4}$, Yanfang Zhang ${ }^{5}$, Jianxun $\mathrm{Qi}^{3}$, George F Gao ${ }^{1,2,3,4,5,6}$ \\ ${ }^{1}$ College of Veterinary Medicine, China Agricultural University, Beijing 100193, China \\ ${ }^{2}$ Research Network of Immunity and Health (RNIH), Beijing Institutes of Life Science, Chinese Academy of Sciences, Beijing \\ 100101, China \\ ${ }^{3}$ CAS Key Laboratory of Pathogenic Microbiology and Immunology, Institute of Microbiology, Chinese Academy of Sciences, \\ Beijing 100101, China \\ ${ }^{4}$ University of Chinese Academy of Sciences, Chinese Academy of Sciences, Beijing 100049, China \\ ${ }^{5}$ Laboratory of Protein Engineering and Vaccines, Tianjin Institute of Industrial Biotechnology, Chinese Academy of Sciences, \\ Tianjin 300308, China \\ ${ }^{6}$ National Institute for Viral Disease Control and Prevention, Chinese Center for Disease Control and Prevention (China CDC), \\ Beijing 102206, China \\ $\triangle$ Correspondence: gaof@im.ac.cn
}

Received May 10, 2013 Accpeted June 1, 2013

\begin{abstract}
Avian influenza A virus continues to pose a global threat with occasional H5N1 human infections, which is emphasized by a recent severe human infection caused by avian-origin H7N9 in China. Luckily these viruses do not transmit efficiently in human populations. With a few amino acid substitutions of the hemagglutinin $\mathrm{H} 5$ protein in the laboratory, two H5 mutants have been shown to obtain an air-borne transmission in a mammalian ferret model. Here in this study one of the mutant H5 proteins developed by Kawaoka's group (VN1203mut) was expressed in a baculovirus system and its receptor-binding properties were assessed. We herein show that the VN1203mut had a dramatically reduced binding affinity for the avian $\alpha 2,3-$ linkage receptor compared to wild type but showed no detectable increase in affinity for the human $\alpha 2,6$-linkage receptor, using Surface Plasmon Resonance techonology. Further, the crystal structures of the VN1203mut and its complexes with either human or avian receptors demonstrate that the VN1203mut binds the human receptor in the same binding manner (cis conformation) as seen for the HAs of previously reported 1957 and 1968 pandemic influenza viruses. Our receptor binding and crystallographic data shown here further confirm that the ability to bind the avian receptor has to decrease for a higher hu-
\end{abstract}

man receptor binding affinity. As the Q226L substitution is shown important for obtaining human receptor binding, we suspect that the newly emerged H7N9 binds human receptor as H7 has a Q226L substitution.

KEYWORDS airborne, transmission, H5, avian influenza, structure, receptor binding

\section{INTRODUCTION}

Recent severe human infections by avian-origin influenza $A$ virus $\mathrm{H} 7 \mathrm{~N} 9$ has again reminded the world of the threat of avian influenza virus to human beings (Gao et al., 2013). Interspecies transmission of highly pathogenic avian influenza (HPAl) virus H5N1 ("bird flu") to humans was first recorded in Hong Kong SAR, China, in 1997 and ever since sporadic cases have been reported worldwide every year (www.who.org). To date, World Health Organization (WHO) data indicate that since 2003, HPAI H5N1 viruses have infected 622 people and killed 371 (as of April 8th, 2013). Luckily, the H5N1 virus has not yet acquired the ability to efficiently transmit among humans.

There are four recorded pandemics in the history, 1918 Spanish flu, 1957 Asian flu, 1968 Hong Kong flu and 2009 pH1N1 pandemic, with a less severe pandemic in 1977, Russian flu (Medina and Garcia-Sastre, 2011). It has been shown that these viruses were linked to avian influenza viruses, with 
some genes derived from avian-origin viruses (Webster et al., 1992). Therefore, elucidation of the underlying mechanism of how avian influenza virus genes adapt to humans is a key point for the scientific community and for the control of influenza virus infection and potential pandemics.

Influenza A virus is an enveloped negative-stranded RNA virus with 8 gene segments (Medina and Garcia-Sastre, 2011). Gene reassortment occurs frequently to produce emerging viruses (Peiris et al., 2007). The surface glycoprotein hemagglutinin (HA) is responsible for virus entry and fusion by binding to the cellular receptor, sialic acid (SA) (Rogers et al., 1983). There are 16 serotypes of HA to date with a newly identified $\mathrm{H} 17$ from bat whose function is distinct (Tong et al., 2012; Sun et al., 2013; Zhu et al., 2013). HA is one of the major genetic traits for interspecies transmission (Neumann et al., 2009). The receptor SAs are usually found in either $\alpha 2,3-$ or $\alpha 2,6$-linkage to galactose (Gal), which determine the host specificity for infection. Avian influenza viruses in general bind $\alpha 2,3-$-linkage receptors whereas human isolates predominantly bind $\alpha 2,6$ linkage receptors. As a mixing vessel, the respiratory tract of swine express both receptors. The binding mechanism and the major amino acids involved in the receptor binding in $\mathrm{HA}$ proteins have been well defined for some HAs, e.g. H1, H2 and H3 (Stevens et al., 2004; Tumpey et al., 2007). However, the H5 HA structural adaptation to the human receptor binding requires further definition (Yamada et al., 2006). It has been shown that experimental changes at position 226 and 228 ( $\mathrm{H} 3$ numbering) in $\mathrm{H} 5$ resulted in the recognition of humantype receptors (Stevens et al., 2006). Yet, it remains unclear whether these amino acid substitutions confer transmission in mammalian models. Recent studies have shown that the ANietnam/1203/2004 H5N1 influenza virus requires four substitutions and reassortment, to become transmissible between ferrets via respiratory droplets (Imai et al., 2012). The four amino acid substitutions in HA identified contain two receptor-binding domain amino acid substitutions, N224K and Q226L, known to change the sialic acid linkage preference to more humanlike $\alpha 2-6$ linkage. The remaining two substitutions are N158D, which disrupts the $\mathrm{N}$-linked glycosylation and T318I in the stalk region, which presumably stabilizes the trimer structure.

Here, we generated the soluble H5 HA protein and its mutant from the influenza virus A/Vietnam/1203/2004 (VN1203 and VN1203mut), which was well studied in the Kawaoka group's study. The structures of the VN1203mut, both in a free form and in complex with avian and human receptor analogues, were determined. We show that the VN1203mut has dramatically reduced binding affinity to avian receptor, but similar affinity to human receptor, compared with the wild type HA. Further structural analysis reveals that the VN1203mut has a wider receptor binding site (RBS), and more importantly, the VN1203mut binds the human receptor in the same pattern (cis conformation) as the HAs of $1957 \mathrm{H} 2$ and $1968 \mathrm{H} 3$ pandemic influenza viruses.

\section{RESULTS AND DISCUSSION}

Receptor binding properties of the wild type H5 (VN1203) vs its mutant (VN1203mut)

The sequences encoding the ectodomain of the wildtype $\mathrm{H} 5$ (VN1203) and its mutant (VN1203mut, containing N158D/ N224K/Q226L/T318I, H3 numbering) were cloned into the pFastbac1 vector and expressed using a baculovirus expression system, as previously described (Zhang et al., 2010; Lu et al., 2012). The receptor binding properties of these two proteins were investigated by Surface Plasmon Resonance (SPR) technology and glycan microarrays. SPR experiments show that the VN1203 preferentially binds avian receptor (affinity of $6 \mu \mathrm{mol} / \mathrm{L})$, with no detectable affinity of the human receptor (Fig. 1). However, the VN1203mut displays a dramatically reduced affinity of avian receptor. There is still no detectable affinity of the human receptor for the VN1203mut. Next, largescale glycan microarray analysis was performed to examine the receptor binding properties of the VN1203 and VN1203mut proteins. This glycan microarray consists of $>600$ glycans, including natural sialosides ( $\alpha 2,3$-linkage, $\alpha 2,6$-linkage, $\alpha 2,8-$ linkage, and mixed linkage) and other glycans that may be relevant to influenza biology. The VN1203 displayed significant avidity to $\alpha 2,3-$ linkage sialylated glycans, while the VN1203mut displayed no obvious avidity to any glycans on the microarray.

\section{Structural basis for receptor-binding changes}

Using X-ray crystallography, we solved the structures of VN1203 mut in its free form and in complex with either avian or human receptor analogs, the sialo-pentasaccharides LSTa and LSTc that are natural sialosides from human milk (Eisen et al., 1997). Crystallographic data collection and refinement statistics are shown in Table 1. Unfortunately we were unable to obtain the structures of VN1203 in complex with avian and human receptor analogs. As the receptor binding site of VN1203 is similar to the previously reported Singapore-H5 (Ha et al., 2001), we used the complex structures of Singapore-H5/LSTa and Singapore-H5/LSTc to compare with our complex structures of VN1203mut/LSTa and VN1203mut/LSTc.

Only the electron density for terminal sialic acid moiety (Sia-1) is observed in the complex structure of VN1203mut with avian receptor analog LSTa. The good quality of the X-ray data and the well-refined statistics from the crystallographic refinement suggest that the poor electron density map of the LSTa is due to low affinity binding and/or the binding of multiple conformations. In this complex, in addition to the conserved hydrogen bond interaction from the base residue $\mathrm{Y} 98$, the sialic acid is stabilized by six hydrogen bonds formed by residues $\mathrm{S} 135$, S136, S137, N186 and E190 (Fig. 2A, Table 2). The hydrogen bond formed between the sialic acid and N186 is mediated by a water molecule. The structure of Singapore-H5 bound to the LSTa revealed that the ligand binds in a trans conformation 
A

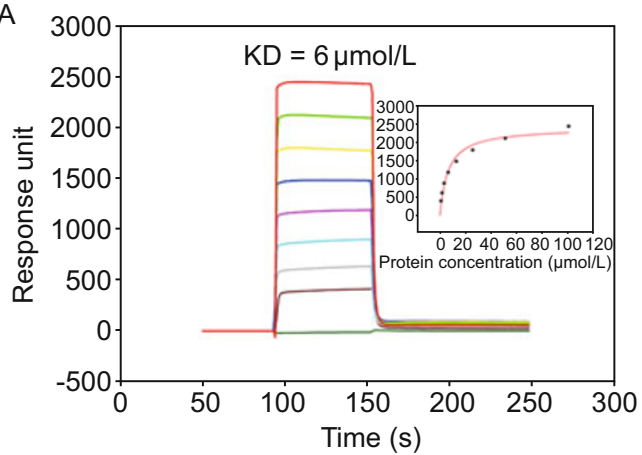

C

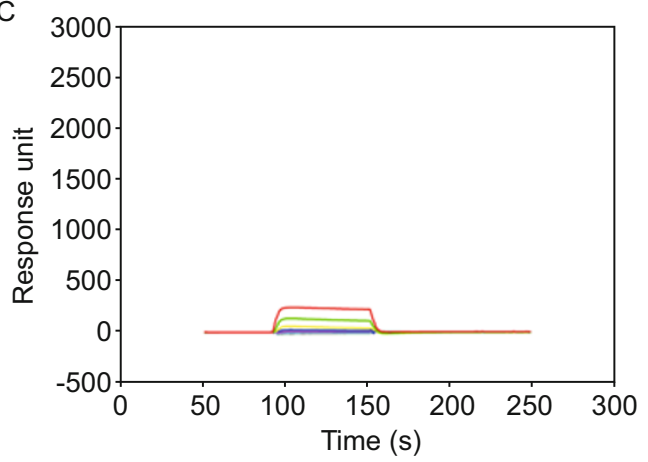

E

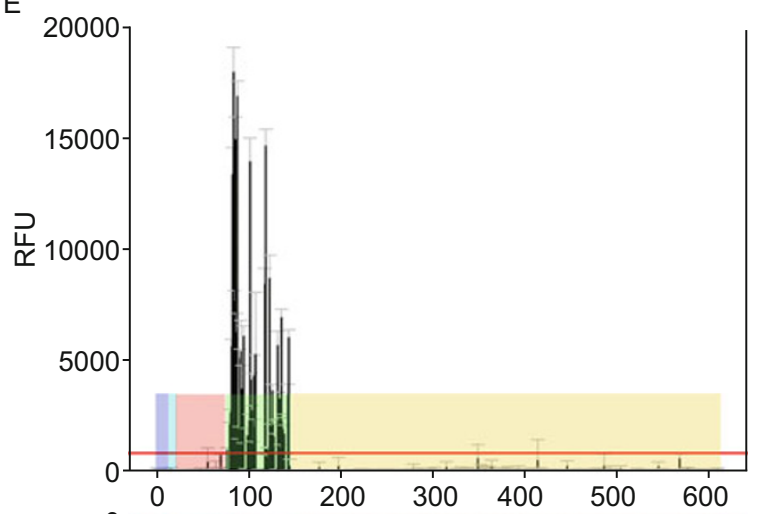

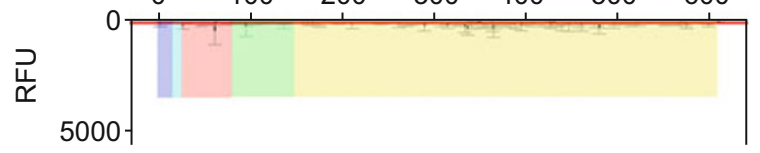

B

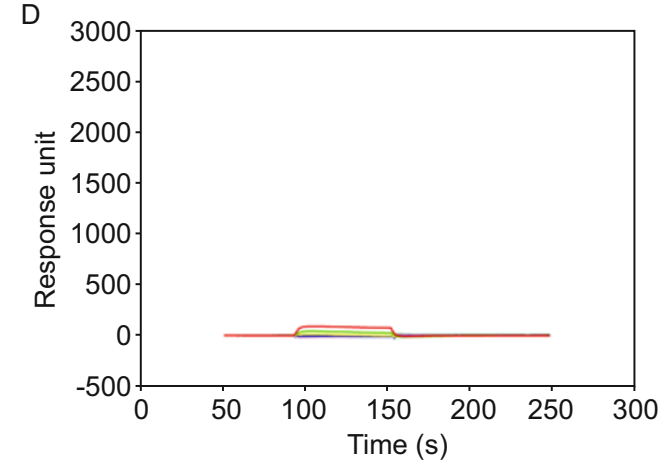

Figure 1. BIAcore ${ }^{\circledR}$ binding properties of the VN1203 and VN1203mut HAs to either $\alpha 2,3$-linked or $\alpha 2,6$-linked sialylglycan receptors and glycan microarray analysis. (A and B) BIAcore ${ }^{\circledR}$ diagram of VN1203 binding to the two receptors, showing strong binding to the $\alpha 2,3$-linked sialylglycan receptor but undetectable binding to the $\alpha 2,6$-linked sialylglycan receptor. (C and D) BIAcore ${ }^{\circledR}$ diagram of VN1203mut binding to the two receptors, showing undetectable binding to both the $\alpha 2,3$-linked sialylglycan and $\alpha 2,6$-linked sialylglycan receptors. (E) Glycan microarray analyses of the VN1203 protein (upper panel) and the VN1203mut protein (lower panel). Binding to different types of glycans on the array is highlighted, where purple represents Neu5Gc, cyan represents $\alpha 2,8$-ligands, pink represents $\alpha 2,6$-ligands, green represents a2,3-ligands, and yellow represents other glycans. The VN1203 protein displayed a good avidity to $\alpha 2,3$-ligands, but the VN1203mut protein showed no obvious avidity to any of the glycans. The standard deviation of the mean value is shown in error bar.

(Fig. 2B). Compared with the VN1203mut/LSTa complex structure, a similar interaction with sialic acid is observed in this complex, and more importantly, the hydrophilic residues Q226 forms one hydrogen bond with the hydrophilic glycosidic oxygen between the terminal sialic acid (Sia-1) and the following sugar (Gal-2). However, the equivalent position 226 of VN1203 mut was substituted with a hydrophobic residue leucine (L), which creates an unfavorable hydrophobic environment for the glycosidic oxygen. Thus, this clash may explain why the VN1203mut displays a dramatically reduced affinity of avian receptor, different from the VN1203 which contains Q226 in the receptor binding site.

The structure of the VN1203mut bound to the human receptor analog LSTc revealed that the ligand binds in a cis conformation (Fig. 2C). In this complex, the sialic acid is stabilized by six hydrogen bonds formed by residues S135, S136, S137, E190 and G228 (Fig. 2C, Table 3). The hydrogen bond formed between the sialic acid and G228 is mediated by a water molecule. The hydrophobic residues L226 forms van der Wall contacts with the non-polar portion around the $\mathrm{C} 6$ atom of Gal-2 through the methyl group. In contrast, only sialic acid is observed in the structure of Singapore-H5 bound to the LSTc, which is similarly seen in the structure of VN1203mut bound to the LSTa. The poor quality of the image of the LSTc may also be due to the low affinity binding and/or the binding of multiple conformations.

Previous studies demonstrate that RBSs of the human and swine influenza virus HAs are larger than those of the avian 
Table 1. Crystallographic data collection and refinement statistics

\begin{tabular}{|c|c|c|c|c|}
\hline & VN1203mut & VN1203mut-LSTa & VN1203mut-LSTc & XJH5 \\
\hline \multicolumn{5}{|l|}{ Data collection } \\
\hline Space group & $\mathrm{P} 2_{1}$ & $\mathrm{P} 2_{1}$ & $\mathrm{P} 2_{1}$ & $\mathrm{P} 3_{1}$ \\
\hline \multicolumn{5}{|l|}{ Cell dimensions } \\
\hline$a, b, c(\AA)$ & $66.76,243.85,71.61$ & $66.68,245.16,68.56$ & $67.26,245.91,69.57$ & $72.05,72.05,365.86$ \\
\hline$\alpha, \beta, \gamma\left({ }^{\circ}\right)$ & $90.00,110.01,90$ & $90.00,113.75,90.00$ & $90.00,112.96,90.00$ & $90,90,120$ \\
\hline Resolution $(\AA)$ & $50-2.50(2.59-2.50)$ & $50-2.50(2.59-2.50)$ & $50-2.40(2.49-2.40)$ & $50-2.60(2.69-2.60)$ \\
\hline$R_{\text {merge }}$ & $0.076(0.644)$ & $0.089(0.640)$ & $0.085(0.513)$ & $0.085(0.478)$ \\
\hline$\| / \sigma l$ & $18.1(2.6)$ & $13.2(2.0)$ & $14.6(3.1)$ & $16.6(3.2)$ \\
\hline Completeness (\%) & $99.6(99.9)$ & $97.7(99.4)$ & $98.3(99.8)$ & $99.9(100.0)$ \\
\hline Redundancy & $4.2(4.3)$ & $3.5(3.5)$ & $3.6(3.8)$ & $4.6(4.6)$ \\
\hline \multicolumn{5}{|l|}{ Refinement } \\
\hline Resolution (Å) & $35.07-2.50$ & $35.40-2.50$ & $34.2-2.40$ & $36.87-2.60$ \\
\hline No. reflections & 73934 & 68621 & 78961 & 64905 \\
\hline$R_{\text {work }} / R_{\text {free }}$ & $0.2151 / 0.2508$ & $0.2229 / 0.2592$ & $0.2370 / 0.2688$ & $0.2332 / 0.2623$ \\
\hline \multicolumn{5}{|l|}{ No. atoms } \\
\hline Protein & 12051 & 12051 & 12023 & 11732 \\
\hline Ligand/ion & 0 & 42 & 67 & 0 \\
\hline Water & 171 & 176 & 283 & 73 \\
\hline \multicolumn{5}{|l|}{$B$-factors } \\
\hline Protein & 60.5 & 62.0 & 66.7 & 74.2 \\
\hline Ligand/ion & 0 & 77.3 & 82.2 & 0 \\
\hline Water & 41.7 & 48.3 & 52.2 & 72.3 \\
\hline \multicolumn{5}{|l|}{ R. m.s. deviations } \\
\hline Bond lengths $(\AA)$ & 0.005 & 0.005 & 0.012 & 0.006 \\
\hline Bond angles $\left({ }^{\circ}\right)$ & 1.083 & 1.103 & 1.558 & 1.024 \\
\hline \multicolumn{5}{|l|}{ Ramachandran analysis } \\
\hline Most favoured (\%) & 86.7 & 86.2 & 84.8 & 85.1 \\
\hline Additional allowed (\%) & 12.3 & 12.7 & 14.3 & 14.4 \\
\hline Generously allowed (\%) & 1 & 1.1 & 1 & 0.5 \\
\hline Disallowed (\%) & 0 & 0 & 0 & 0 \\
\hline
\end{tabular}

influenza virus HAs (Stevens et al., 2004). Comparison of the previously reported VN1203 HA (Stevens et al., 2006), the VN1203mut, the 1957 Singapore human $\mathrm{H} 2(57 \mathrm{H} 2)$ and the 1968 Hong Kong human $\mathrm{H} 3(68 \mathrm{H} 3)$ was made to see if the Q226L substitutions alone can convert avian type HA into a humanlike HA. The distance between the 130-loop and the 220-loop, which form two sides of the receptor binding site, is greater by $\sim 1.5 \AA$ in the VN1203mut structure than in the VN1203 structure (Fig. 3A). The distances between the 130-loop and 220loop are comparable in the VN1203mut, $57 \mathrm{H} 2$ and $68 \mathrm{H} 3$ structures (Fig. 3B). However, the VN1203mut does not display as strong a binding affinity for the human receptor, as $57 \mathrm{H} 2$ and $68 \mathrm{H} 3$ do. Our structural comparisons showed a $\sim 3 \AA$ displacement in the VN1203mut/LSTc complex relative to the $57 \mathrm{H} 2$ /
LSTc and 68H3/LSTc complex (Fig. 3C and 3D), resulting in fewer contacts with the RBS. In total, the VN1203mut binds the human receptor in the same pattern (cis conformation) as the $57 \mathrm{H} 2$ and $68 \mathrm{H} 3$ do.

Thermostability of VN1203mut and contribution of the 158-160 glycosylation to the receptor binding

In addition to the Q226L substitution, three other amino acid substitutions (N158D, N224K and T318I) were also introduced into the VN1203mut (Fig. 4A). The N158D substitution, which results in the loss of a glycosylation site on the head of the $\mathrm{HA}$ close to the receptor binding site, enhances H5N1 virus binding to the a2,6-linked human receptor (Neumann et al., 
A

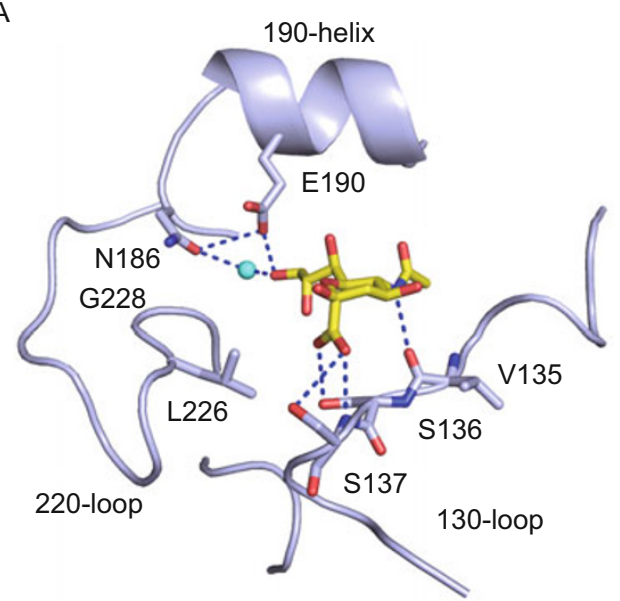

B

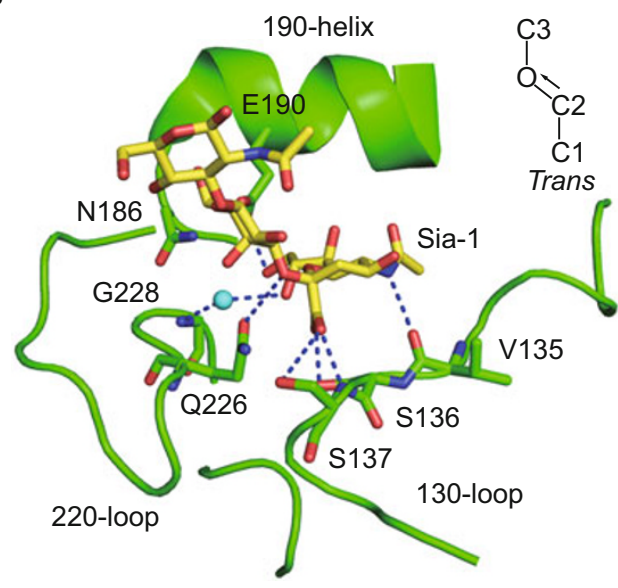

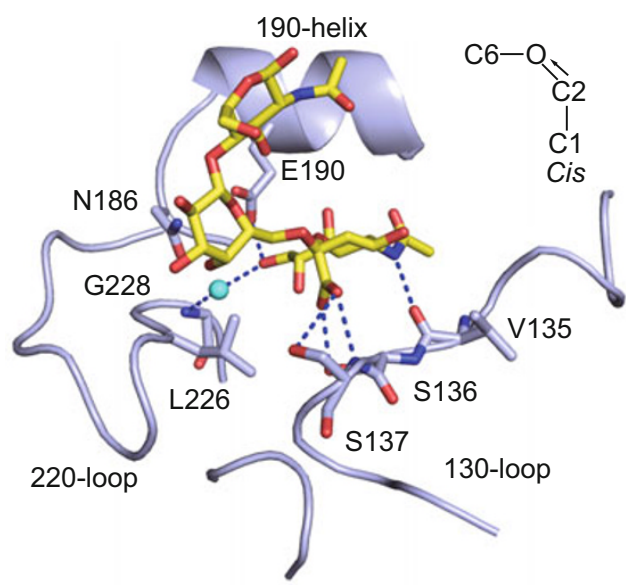

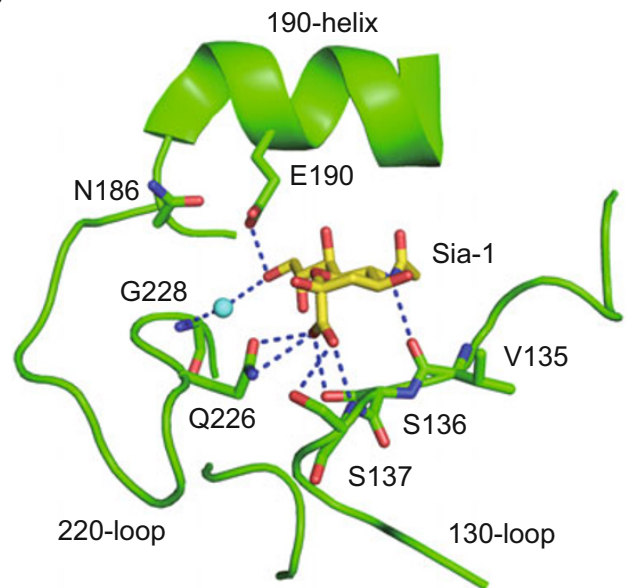

Figure 2. Interaction of the VN1203mut HA with either avian or human receptor analogues and comparison with previously reported Singarpore-H5/receptor complexes. The three secondary structural elements of the binding site (i.e., the 130-loop, 190-helix and 220-loop) are labeled in ribbon representation, together with selected residues in stick representation. The hydrogen bonds are shown as dashed lines. The VN1203mut is colored in light blue, and the Singarpore-H5 is colored in green. The glycans are colored in yellow. (A and C) VN1203mut with the avian receptor analog LSTa $(\alpha 2,3)$ pentasaccharide $(A)$ or human receptor analog LSTc ( $\alpha 2,6)$ pentasaccharide (C) bound. LSTc binds in a cis conformation. (B and D) Singarpore-H5 with the avian receptor analogue LSTa (B) or the human receptor analogue LSTc (D) bound. LSTa binds in a trans conformation.

Table 2. Hydrogen bond interactions between VN1203mut and LSTa

\begin{tabular}{lllll}
\hline VN1203mut/a2,3 & Protein & & Average bond distance $(\AA)$ \\
\hline & O1A & OG & $136 \mathrm{~S}$ & 3.10 \\
& O1B & N & $137 \mathrm{~S}$ & 3.15 \\
SIA-1 & O1B & OH & $137 \mathrm{~S}$ & 3.50 \\
& N5 & O & $135 \mathrm{~V}$ & 2.90 \\
& O9 & OH & $98 \mathrm{Y}$ & 3.15 \\
& O9 & OE1 & $190 \mathrm{E}$ & 2.47 \\
& O9 & OD1 & $186 \mathrm{~N}$ & Mediated by a water molecule \\
\hline
\end{tabular}

2012). How this glycosylation site affects the receptor binding property remains an intriguing question. However, in the previ- ously reported VN1203 structure and other H5 HA structures, no glycan was observed in this glycosylation site. Here we 
Table 3. Hydrogen bond interactions between VN1203mut and LSTC

\begin{tabular}{lllll}
\hline VN1203mut /a2,6 & Protein & & Average bond distance $(\AA)$ \\
\hline & O1A & OG & $136 \mathrm{~S}$ & 2.87 \\
& O1B & N & $137 \mathrm{~S}$ & 3.14 \\
& O1B & OH & $137 \mathrm{~S}$ & 3.50 \\
SIA-1 & N5 & O & $135 \mathrm{~V}$ & 2.93 \\
& O9 & OE1 & $190 \mathrm{E}$ & 2.69 \\
& O9 & OH & $98 \mathrm{Y}$ & 2.79 \\
& O9 & N & G228 & Mediated by a water molecule \\
\hline
\end{tabular}

A

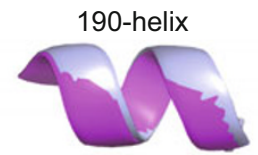

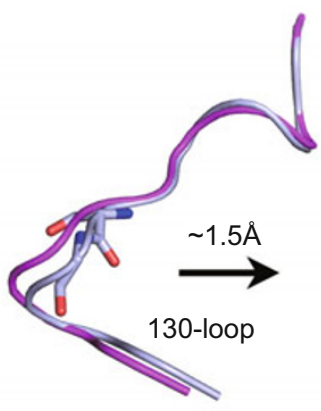

C

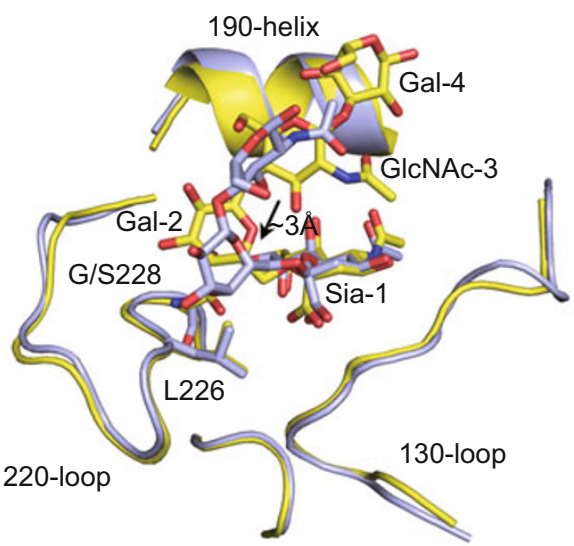

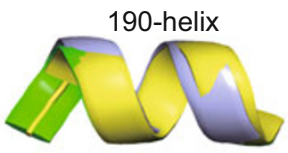

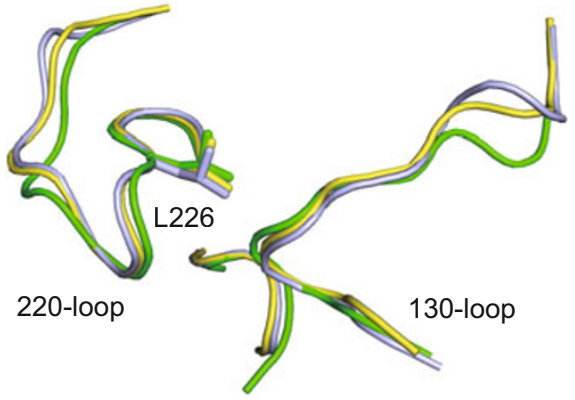

D

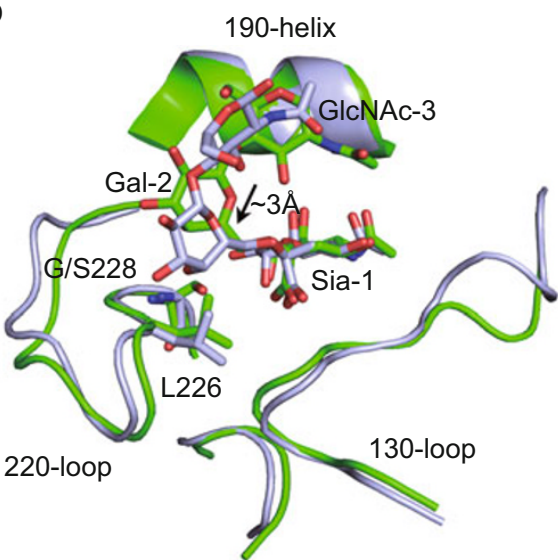

Figure 3. Comparison of VN1203, VN1203mut, human H2/H3 and their HA/LSTc complex structures. (A) Comparison of receptor binding sites between previously reported VN1203 (magenta, PDB code: 2FK0) and VN1203mut (light blue). The receptor binding site of VN1203mut is $\sim 1.5 \AA$ wider than that of VN1203. (B) Comparison of the receptor binding sites between VN1203mut (light blue), 57H2 (yellow) and 68H3 (green). VN1203mut has a similar wide receptor binding site compared to 57H2 (PDB code: 2WR7) and 68H3 (PDB code: 2YPG). (C and D) Comparison of the VN1203mut/LSTc (light blue), 57H2/LSTc (yellow) and 68H3/LSTc (green) complexes. The glycans are displaced away from the receptor binding site by $\sim 3 \AA$ in the VN1203mut/LSTc complex relative to those in the $57 \mathrm{H} 2 / \mathrm{LSTC}$ and $68 \mathrm{H} 3 /$ LSTc complexes.

show the first structural evidence that this glycosylation site can be glycosylated (Fig. 4B), by solving the structure of the $\mathrm{HA}$ of an H5N1 virus isolate (A/Xinjiang/1/2006, XJH5), which was also expressed in a baculovirus system. Based on the structures of VN1203mut/LSTc and this XJH5, we propose a model with manually extended mammalian sugars to elucidate how this glycosylation site affects the receptor binding property (Fig. 4C). As shown in the model, the orientation of the sugars 

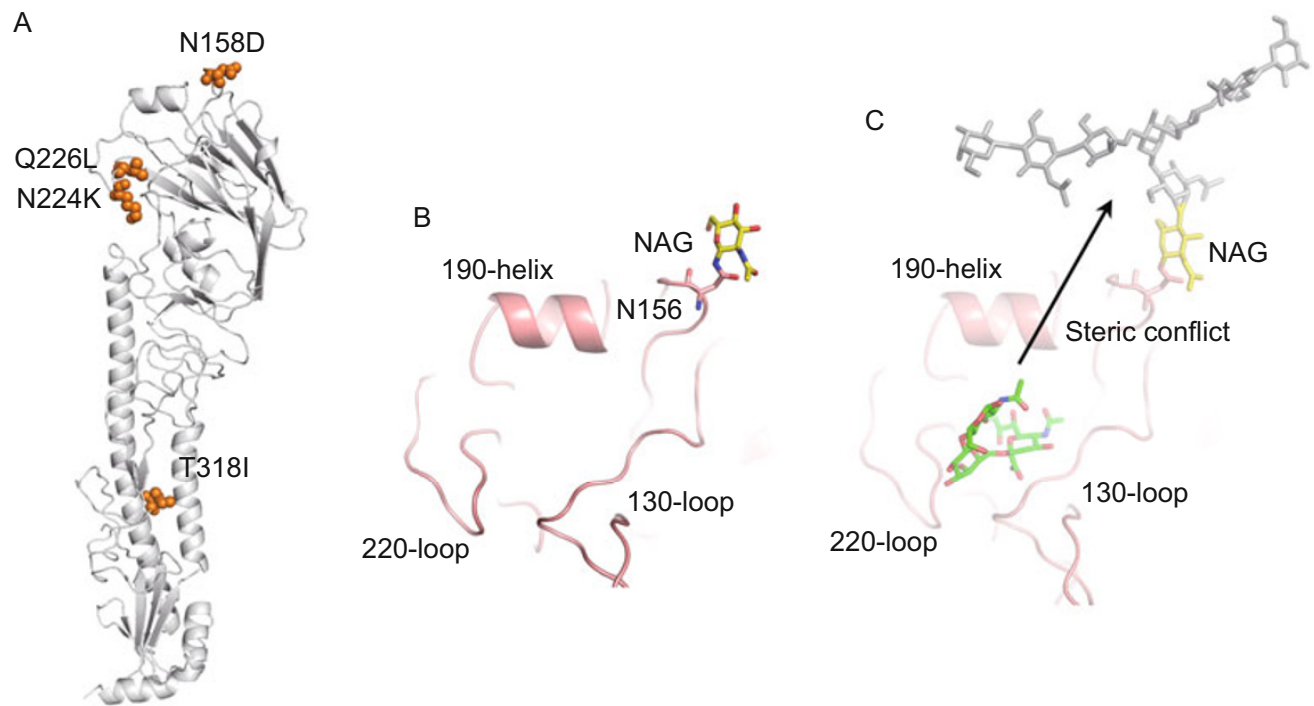

Figure 4. Structural evidence for glycosylation at 150 loop. (A) Cartoon presentation of the four amino acid substitutions (N158D/ N224K/Q226L/T318I) in the VN1203mut structure. (B) Glycosylation at 150 loop in the XJH5 structure. The residue N158 and the glycan NAG are shown in stick representation. (C) A model with manually added mammalian sugars to elucidate how this glycosylation site affects the receptor binding property. The mammalian sugars can make steric conflict with the $\alpha-2,6$ glycan receptor, as the orientation of the glycan of the $\alpha-2,6$ glycan receptor goes towards the mammalian sugars.
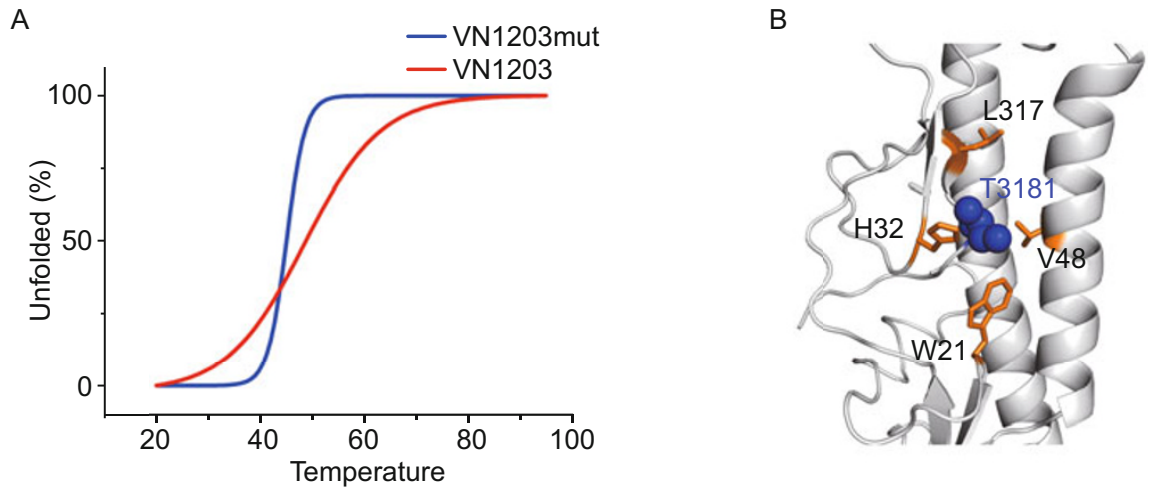

Figure 5. Higher thermostability of VN1203mut compared to VN1203. (A) Thermostability analyses of the VN1203 and VN1203mut proteins. Temperature-dependent CD spectroscopic experiments revealed that the VN1203mut protein has a higher thermostability than VN1203 before $45^{\circ} \mathrm{C}$. (B) Structural basis of the different thermostabilities of the VN1203 and VN1203mut proteins. The three residue substitutions (N158D/N224K/Q226L) appear to be irrelevant to the thermostability. The T318I substitution seems to stabilize the hydrophobic core near the cleavage site, which might preclude the conformation change of fusion peptide.

of LSTc folds back and goes towards the 190-helix, and thus, the mammalian sugars can result in steric conflict with the a2,6-linked human receptors which should have a long sugar chain. Otherwise, the N224K substitution appears to have no affect on the receptor binding.

Temperature-dependent circular dichroism (CD) spectroscopic experiments revealed that the VN1203mut has a higher thermostability than wild type VN1203 before $45^{\circ} \mathrm{C}$ (Fig. 5A). Moreover, the VN1203mut has a more transient denaturation state than the VN1203 (Fig. 5A). These differences might be due to the $\mathrm{T} 318 \mathrm{I}$ substitution, as the other three residue sub- stitutions (N158D/N224K/Q226L) converge on the receptor binding site and appear to be irrelevant to the thermostability. Structure analysis revealed that the residue 318 is surrounded by hydrophobic residues (Fig. 5B), and a threonine-to-isoleucine substitution could increase the stability of the hydrophobic core near the cleavage site. This stabilization might preclude the conformation change of the fusion peptide. Other amino acid changes elsewhere in the virus may also be critical for enabling the $\mathrm{H} 5 \mathrm{~N} 1$ virus to transmit between humans. For example, Herfst et al. (2012) introduced E627K into the PB2 protein (Hatta et al., 2001), together with the two substitutions 
introduced by reverse genetics and two acquired upon ferret passage in the $\mathrm{A} /$ Indonesia/5/2005 virus, to generate a H5N1 virus that is transmissible among ferrets (Herfst et al., 2012).

In conclusion, here we have revealed the structural and biophysical basis of the airborne transmissibility of an avian influenza hemagglutinin H5. Clearly, it demonstrates that reduction to the avian-type receptor binding is a prerequisite for transmissibility. This particular $\mathrm{H} 5$ mutant hemagglutinin binds the human $\alpha 2,6$-linked receptor in the same binding pattern (cis conformation) as seen for the HAs of previously reported 1957 and 1968 pandemic influenza viruses, which warrants further assessment in the future in order to prevent any possible pandemic caused by H5N1 subtype of avain influenza virus. During the preparation of this manuscript, two similar results have been reported in top journals (Xiong et al., 2013; Zhang et al., 2013), indicating this research is really a hot area.

\section{MATERIALS AND METHODS}

\section{Gene cloning, protein expression and purification}

Highly stable and pure H5 HA proteins were prepared using previously established methods (Zhang et al., 2010), with slight modifications, using the Bac-to-Bac baculovirus expression system (Invitrogen). The sequences encoding the ectodomain of HA from AViet nam/1203/2004 (VN1203) or A/Xinjiang/1/2006 (XJH5) were cloned into the baculovirus transfer vector pFastBac1 (Invitrogen) respectively, with a GP67 signal peptide at the $\mathrm{N}$-terminus, a thrombin cleavage site, a trimerizing sequence, and a $\mathrm{His}_{6}$ tag at the C-terminus. The VN1203mut was generated by site-directed mutagenesis to change the 4 amino acids N224K, Q226L, N158D and T318I as pointed out by Kawaoka et al. (Imai et al., 2012). The recombinant baculoviruses were prepared based on the manufacturer's protocol (Invitrogen). The HA proteins were obtained from infected $\mathrm{Hi} 5$ insect cells using previously reported purification methods (Zhang et al., 2010). The collected protein fractions were concentrated to $10 \mathrm{mg} / \mathrm{mL}$ for further use.

\section{Crystallization, data collection and structure determination}

Crystallization conditions were screened using the sitting-drop vapor diffusion method with commercial kits. For VN1203mut, the crystals were obtained in the crystallization conditions of $0.1 \mathrm{~mol} / \mathrm{L}$ sodium cacodylate $\mathrm{pH} 6.0,15 \% \mathrm{w} / \mathrm{V}$ PEG 4000 at $18^{\circ} \mathrm{C}$. For receptor analog complexes, crystals were soaked in a reservoir solution containing $8 \mathrm{mmol} / \mathrm{L} \mathrm{LSTa}$ or LSTc for $4 \mathrm{~h}$ upon the crystallization condition. The XJH5 crystals were obtained with a condition of 10\% PEG 6000, 5\% MPD, $0.1 \mathrm{~mol} / \mathrm{L}$ Hepes, $\mathrm{pH} 7.5$ at $20^{\circ} \mathrm{C}$. All crystals were flash-cooled in liquid nitrogen after a brief soaking in reservoir solution with the addition of $17 \%(v / v)$ glycerol. X-ray diffraction data were collected at $100^{\circ} \mathrm{K}$ at Shanghai Synchrotron Radiation Facility (SSRF) beamline BL17U. These data were processed and scaled using the HKL-2000 program (Vaguine et al., 1999). Data collection and processing statistics are summarized in Table 1. The structures were solved by the molecular replacement (MR) method using Phaser (Read, 2001) from the CCP4 program suite (Collaborative Computational Project, 1994), with the structure of the H5 HA from ANietNam/1203/2004 (PDB code 2fk0) as the search model. Model building and refinement were performed using the COOT (Emsley and Cowtan, 2004) and REFMAC5 (Murshu- dov et al., 1997) programs, respectively. The stereochemical quality of the final models was assessed with the program PROCHECK (Morris et al., 1992). The structures of VN1203mut, VN1203mut/LSTa, and VN1203mut/LSTc and XJH5 are deposited to Protein Data Bank as $4 \mathrm{KDM}, 4 \mathrm{KDN}, 4 \mathrm{KDO}$ and $4 \mathrm{KDQ}$.

\section{Surface plamon resonance (SPR) analysis}

The affinity and kinetics of the binding of soluble VN1203 and VN1203 mut to receptor analogs were measured at $25^{\circ} \mathrm{C}$ on a BIAcore ${ }^{\circledR}$ 3000 using streptavidin chips (SA chips, Biacore ${ }^{\circledR}$ ) by SPR. PBST buffer (phosphate-buffered saline containing $0.005 \%$ Tween 20) was used as the kinetics analysis buffer. Two biotinylated receptor analogs, the $a-2,6$ glycans (6'S-Di-LN: Neu5Aca2-6[Galb1-4GlcNAcb1-3]2bSpNH-LC-LC-Biotin) and the $\alpha-2,3$ glycans (3'S-Di-LN: Neu5Aca23[Galb1-4GlcNAcb1-3]2b-SpNH-LC-LC-Biotin) were kindly provided by the Consortium for Functional Glycomics. The $\alpha-2,6$ glycans and $\alpha-2,3$ glycans were immobilized on the CM5 chip with 400 response units (RU). Trypsin-treated HAs were purified by gel filtration using PBST buffer as running buffer and serially diluted to concentrations ranging from $0.78-100 \mu \mathrm{mol} / \mathrm{L}$. HA protein was then flowed through the chip and the RU was measured. The sensor surface was regenerated with $10 \mathrm{mmol} / \mathrm{L} \mathrm{NaOH}$ at the end of each cycle. The data were analyzed using BIAevaluation ${ }^{\circledR}$ software and fitted to a 1:1 binding model using HA monomer as the calculating molecular weight.

\section{Glycan microarray analysis}

Glycan microarray analysis was performed using $20 \mathrm{mmol} / \mathrm{L}$ Tris- $\mathrm{HCl}$ (pH 8.0) and $150 \mathrm{mmol} / \mathrm{L} \mathrm{NaCl}$ as the running buffer. Glycan microarray printing and recombinant $\mathrm{HA}$ analyses were carried out as has previously been described (Sun et al., 2013). Briefly, the analyses were performed by applying the protein to the array at $200 \mu \mathrm{g} / \mathrm{mL}$ and detection in the second step with an anti-His antibody labeled with Alexa488. Version 5.0 of the printed array consists of 610 glycans in replicates of six. The highest and lowest point from each set of six replicates was removed, so the average is of four values rather than six. This work was carried out in the Consortium for Functional Glycomics by Drs Jamie Heimburg-Molinaro and David F Smith.

\section{Thermostability measurements using circular dichroism (CD)}

The thermostability of VN1203 and VN1203mut was tested by CD spectroscopy (Petersen et al., 2009). Trypsin treated HAs were purified by gel filtration using running buffer of $(20 \mathrm{mmol} / \mathrm{L}$ Tris- $\mathrm{HCl}$, $50 \mathrm{mmol} / \mathrm{L} \mathrm{NaCl}, \mathrm{pH} 8.0$ ) and diluted into $0.2 \mathrm{mg} / \mathrm{mL}$. CD spectra were measured on a Chirascan spectrometer (Applied Photophysics) using a thermostatically controlled cuvette at temperatures between $20^{\circ} \mathrm{C}$ and $94^{\circ} \mathrm{C}$. Far-UV spectra at $218 \mathrm{~nm}$ were collected with a $5 \mathrm{~s} /$ point signal averaging and were the accumulation of 5 individual scans; thermal denaturation curves were determined by monitoring the $C D$ value given as the mean residue ellipticity $\theta_{218}$ at temperature intervals of $0.5^{\circ} \mathrm{C}$ at a rate of $1^{\circ} \mathrm{C} / \mathrm{min}$. The temperature of the protein solution was continuously measured with a sensitive thermocouple. The fraction of unfolded protein was calculated from the mean residue ellipticity $(\theta)$ by the standard method. Fraction unfolded $(\%)$ is expressed as $(\theta-$ $\left.\theta_{N}\right) /\left(\theta_{U}-\theta_{N}\right)$, where $\theta_{N}$ and $\theta_{U}$ are the mean residue ellipticity values in the fully folded and fully unfolded states, respectively. The denaturation curves were generated by linear fitting with the OriginPro 8.0 program 
(OriginLab) as described previously (Zhang et al., 2011). The midpoint of thermal denaturation temperature $\left(T_{\mathrm{m}}\right)$ for each complex was calculated by taking the first derivative of the fitting data and identifying the inflection point, which represents the $T_{\mathrm{m}}$ for each $\mathrm{HA}$ (Webb et al., 2004).

\section{ACKNOWLEDGEMENTS}

This work was supported by the National Basic Research Program (973 Program) (No. 2011CB504703) and the National Natural Science Foundation of China (Grant No. 81290342). G.F.G. is a leading principal investigator of the NSFC Innovative Research Group (Grant No. 81021003). We wish to acknowledge the Consortium for Functional Glycomics for glycan array analysis and synthetic glycans used in binding assays (NIH Grant GM62116). Assistance by the staff at Shanghai Synchrotron Radiation Facility (SSRF-beamline 17U) is acknowledged.

\section{COMPLIANCE WITH ETHICS GUIDELINES}

Xishan Lu, Yi Shi, Wei Zhang, Yanfang Zhang, Jianxun Qi and George F Gao declare that they have no conflict of interest.

This article does not contain any studies with human or animal subjects performed by the any of the authors.

\section{REFERENCES}

Collaborative Computational Project, N. (1994). The CCP4 suite: programs for protein crystallography. Acta Crystallogr D Biol Crystallogr 50, 760-763.

Eisen, M.B., Sabesan, S., Skehel, J.J., and Wiley, D.C. (1997). Binding of the influenza A virus to cell-surface receptors: Structures of five hemagglutinin-sialyloligosaccharide complexes determined by $\mathrm{x}$-ray crystallography. Virology 232, 19-31.

Emsley, P., and Cowtan, K. (2004). Coot: model-building tools for molecular graphics. Acta Crystallogr D Biol Crystallogr 60, 2126-2132.

Gao, R., Cao, B., Hu, Y., Feng, Z., Wang, D., Hu, W., Chen, J., Jie, Z., Qu, H., Xu, K., et al. (2013). Human infection with a novel avianorigin influenza A (H7N9) virus. N Engl J Med 368, 1888-1897.

Ha, Y., Stevens, D.J., Skehel, J.J., and Wiley, D.C. (2001). X-ray structures of $\mathrm{H} 5$ avian and $\mathrm{H} 9$ swine influenza virus hemagglutinins bound to avian and human receptor analogs. Proc Natl Acad Sci U S A 98, 11181-11186.

Hatta, M., Gao, P., Halfmann, P., and Kawaoka, Y. (2001). Molecular basis for high virulence of Hong Kong H5N1 influenza A viruses. Science 293, 1840-1842.

Herfst, S., Schrauwen, E.J., Linster, M., Chutinimitkul, S., de Wit, E., Munster, V.J., Sorrell, E.M., Bestebroer, T.M., Burke, D.F., Smith, D.J., et al. (2012). Airborne transmission of influenza A/H5N1 virus between ferrets. Science 336, 1534-1541.

Imai, M., Watanabe, T., Hatta, M., Das, S.C., Ozawa, M., Shinya, K., Zhong, G., Hanson, A., Katsura, H., Watanabe, S., et al. (2012). Experimental adaptation of an influenza $\mathrm{H} 5 \mathrm{HA}$ confers respiratory droplet transmission to a reassortant $\mathrm{H} 5 \mathrm{HA} / \mathrm{H} 1 \mathrm{~N} 1$ virus in ferrets. Nature 486, 420-428.

Lu, X., Shi, Y., Gao, F., Xiao, H., Wang, M., Qi, J., and Gao, G.F. (2012). Insights into avian influenza virus pathogenicity: the hemagglutinin precursor $\mathrm{HAO}$ of subtype $\mathrm{H} 16$ has an alpha-helix structure in Its cleavage site with inefficient HA1/HA2 cleavage. J Virol 86, 12861 12870.

Medina, R.A., and Garcia-Sastre, A. (2011). Influenza A viruses: new research developments. Nat Rev Microbiol 9, 590-603.

Morris, A.L., MacArthur, M.W., Hutchinson, E.G., and Thornton, J.M. (1992). Stereochemical quality of protein structure coordinates. Proteins 12, 345-364.

Murshudov, G.N., Vagin, A.A., and Dodson, E.J. (1997). Refinement of macromolecular structures by the maximum-likelihood method. Acta Crystallogr D Biol Crystallogr 53, 240-255.

Neumann, G., Macken, C.A., Karasin, A.I., Fouchier, R.A., and Kawaoka, Y. (2012). Egyptian H5N1 influenza viruses-cause for concern? PLoS Pathog 8, e1002932.

Neumann, G., Noda, T., and Kawaoka, Y. (2009). Emergence and pandemic potential of swine-origin H1N1 influenza virus. Nature 459, 931-939.

Peiris, J.S., de Jong, M.D., and Guan, Y. (2007). Avian influenza virus (H5N1): a threat to human health. Clin Microbiol Rev 20, 243-267.

Petersen, J., Wurzbacher, S.J., Williamson, N.A., Ramarathinam, S.H., Reid, H.H., Nair, A.K., Zhao, A.Y., Nastovska, R., Rudge, G., Rossjohn, J., et al. (2009). Phosphorylated self-peptides alter human leukocyte antigen class I-restricted antigen presentation and generate tumor-specific epitopes. Proc Natl Acad Sci U S A 106, 2776-2781.

Read, R.J. (2001). Pushing the boundaries of molecular replacement with maximum likelihood. Acta Crystallogr D Biol Crystallogr 57, 1373-1382.

Rogers, G.N., Pritchett, T.J., Lane, J.L., and Paulson, J.C. (1983). Differential sensitivity of human, avian, and equine influenza $A$ viruses to a glycoprotein inhibitor of infection: selection of receptor specific variants. Virology 131, 394-408.

Stevens, J., Blixt, O., Tumpey, T.M., Taubenberger, J.K., Paulson, J.C., and Wilson, I.A. (2006). Structure and receptor specificity of the hemagglutinin from an H5N1 influenza virus. Science 312, 404-410.

Stevens, J., Corper, A.L., Basler, C.F., Taubenberger, J.K., Palese, P., and Wilson, I.A. (2004). Structure of the uncleaved human H1 hemagglutinin from the extinct 1918 influenza virus. Science 303, 1866-1870.

Sun, X., Shi, Y., Lu, X., He, J., Gao, F., Yan, J., Qi, J., and Gao, G.F. (2013). Bat-derived influenza hemagglutinin h17 does not bind canonical avian or human receptors and most likely uses a unique entry mechanism. Cell Rep 3, 769-778.

Tong, S., Li, Y., Rivailler, P., Conrardy, C., Castillo, D.A., Chen, L.M., Recuenco, S., Ellison, J.A., Davis, C.T., York, I.A., et al. (2012). A distinct lineage of influenza A virus from bats. Proc Natl Acad Sci U S A 109, 4269-4274.

Tumpey, T.M., Maines, T.R., Van Hoeven, N., Glaser, L., Solorzano, A., Pappas, C., Cox, N.J., Swayne, D.E., Palese, P., Katz, J.M., et al. (2007). A two-amino acid change in the hemagglutinin of the 1918 influenza virus abolishes transmission. Science 315, 655-659.

Vaguine, A.A., Richelle, J., and Wodak, S.J. (1999). SFCHECK: a unified set of procedures for evaluating the quality of macromolecular structure-factor data and their agreement with the atomic model. Acta Crystallogr D Biol Crystallogr 55, 191-205.

Webb, A.I., Dunstone, M.A., Chen, W., Aguilar, M.I., Chen, Q., Jackson, H., Chang, L., Kjer-Nielsen, L., Beddoe, T., McCluskey, J., et al. (2004). Functional and structural characteristics of NY-ESO- 
1-related HLA A2-restricted epitopes and the design of a novel immunogenic analogue. J Biol Chem 279, 23438-23446.

Webster, R.G., Bean, W.J., Gorman, O.T., Chambers, T.M., and Kawaoka, Y. (1992). Evolution and ecology of influenza A viruses. Microbiol Rev 56, 152-179.

Xiong, X., Coombs, P.J., Martin, S.R., Liu, J., Xiao, H., McCauley, J.W., Locher, K., Walker, P.A., Collins, P.J., Kawaoka, Y., et al. (2013). Receptor binding by a ferret-transmissible $\mathrm{H} 5$ avian influenza virus. Nature 497, 392-396.

Yamada, S., Suzuki, Y., Suzuki, T., Le, M.Q., Nidom, C.A., SakaiTagawa, Y., Muramoto, Y., Ito, M., Kiso, M., Horimoto, T., et al. (2006). Haemagglutinin mutations responsible for the binding of H5N1 influenza A viruses to human-type receptors. Nature 444, 378-382.

Zhang, N., Qi, J., Feng, S., Gao, F., Liu, J., Pan, X., Chen, R., Li, Q., Chen, Z., Li, X., et al. (2011). Crystal structure of swine major histo- compatibility complex class I SLA-1 0401 and identification of 2009 pandemic swine-origin influenza A H1N1 virus cytotoxic T lymphocyte epitope peptides. J Virol 85, 11709-11724.

Zhang, W., Qi, J., Shi, Y., Li, Q., Gao, F., Sun, Y., Lu, X., Lu, Q., Vavricka, C.J., Liu, D., et al. (2010). Crystal structure of the swineorigin A (H1N1)-2009 influenza A virus hemagglutinin (HA) reveals similar antigenicity to that of the 1918 pandemic virus. Protein Cell 1 , 459-467.

Zhang, W., Shi, Y., Lu, X., Shu, Y., Qi, J., and Gao, G.F. (2013). An Airborne Transmissible Avian Influenza H5 Hemagglutinin Seen at the Atomic Level. Science. (In Press).

Zhu, X., Yu, W., McBride, R., Li, Y., Chen, L.M., Donis, R.O., Tong, S., Paulson, J.C., and Wilson, I.A. (2013). Hemagglutinin homologue from $\mathrm{H} 17 \mathrm{~N} 10$ bat influenza virus exhibits divergent receptor-binding and $\mathrm{pH}$-dependent fusion activities. Proc Natl Acad Sci U S A 110, 1458-1463. 